\section{Linearity improvement in biquadratic transconductor-C filters}

\section{A. Tajalli and Y. Leblebici}

A new technique for improving the linearity performance in biquadratic transconductor-C filters is presented. This improvement has been achieved by applying some modifications to the filter topology which considerably relax the linearity requirement on transconductor circuits Using very simple transconductors, $30 \mathrm{~dB}$ improvement in total harmonic distortion compared to the conventional approach has been observed.

Introduction: High-performance and high-frequency filters are essential building blocks in many applications such as modern telecommunication systems [1]. Transconductor-C topology offers a very good alternative for implementing high-frequency filters, however, they generally suffer from weak linearity performance $[1,2]$. This is mainly due to the limited linearity performance of the transconductors used as basic building blocks in this type of filters. Fig. 1 $a$ shows the conventional topology of a lowpass biquadratic transconductor-C filter [2]. In this topology, the input differential voltage is applied to the first $\mathrm{G}_{\mathrm{m}}$ cell, converted to current, and the subsequent circuitry is used to implement a second-order lowpass filter. Therefore, the linearity of the filter directly depends on the linearity of the transconductors: to realise a linear filter, the transconductors must show a very good linearity for the intended voltage swing at their inputs. A number of circuit topologies have been proposed to implement a linear transconductor [2-4]. However, these topologies are generally very complex and can achieve only a moderate linearity performance. Meanwhile, the complexity of the transconductor can significantly increase the power consumption of the filter as well as the total circuit noise. The circuit complexity can even prevent their effective usage in high-frequency applications.
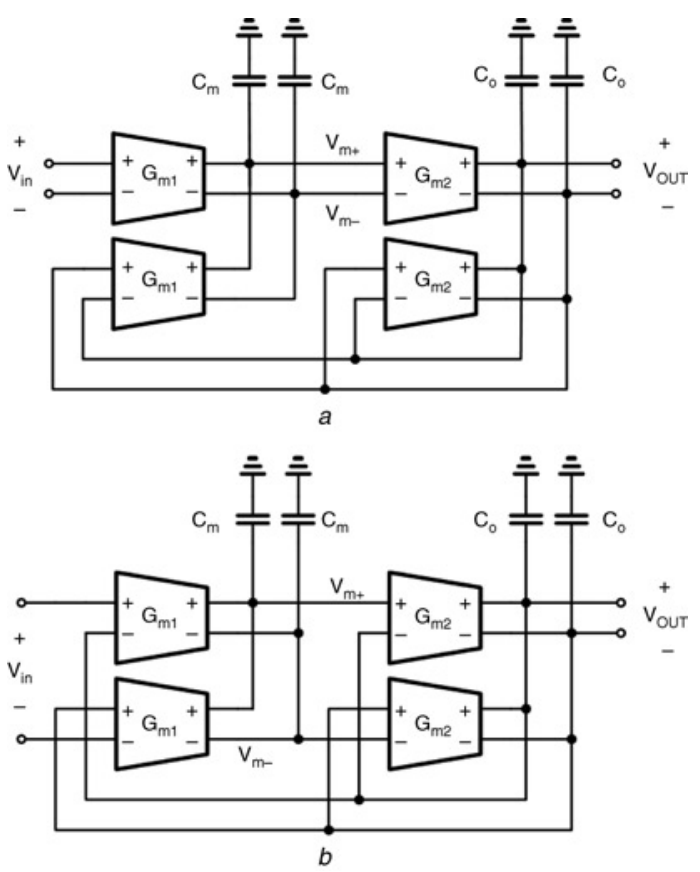

Fig. 1 Biquadratic transconductor filter topologies

$a$ Conventional topology

$b$ Proposed topology

In the following, a modified biquadratic filter topology is be introduced that is inherently more linear than the conventional biquadratic topologies. Therefore, it does not impose any special requirement on the transconductors and hence it can be applied for implementing very linear filters using simple and hence low-cost transconductor circuits.

Proposed topology: The conventional biquadratic filter shown in Fig. $1 a$ uses four differential transconductors and two pairs of capacitors to implement the intended frequency transfer function. Here, all signals are differential and the output current of each transconductor is proportional to the input differential voltage as: $I_{\text {out }}=G_{m} \cdot\left(V_{i+}-\right.$
$V_{i-}$ ). Hence, it is very important that the $G_{m}$ value remains constant for the entire differential voltage swing at the corresponding input node. Now, consider the topology shown in Fig. $1 b$ in which the negative inputs of the transconductors with common outputs have been exchanged compared to the Fig. $1 a$. If $\mathrm{I}_{\mathrm{CO}}$ and $\mathrm{I}_{\mathrm{CM}}$ represent the total current delivered to $\mathrm{C}_{\mathrm{O}}$ and $\mathrm{C}_{\mathrm{M}}$, respectively, then:

$$
\begin{aligned}
I_{C M} & =G_{m 1}\left(V_{i+}-V_{i-}\right)+G_{m 1}\left(V_{o-}-V_{o+}\right) \\
& =G_{m 1}\left(V_{i+}-V_{o+}\right)+G_{m 1}\left(V_{o-}-V_{i-}\right)
\end{aligned}
$$

and similarly:

$$
I_{C O}=G_{m 2}\left(V_{m+}-V_{o+}\right)+G_{m 2}\left(V_{o-}-V_{m-}\right)
$$

Therefore, the current delivered to each capacitor remains unchanged. Similarly, both filter topologies have the same cutoff frequency $\left(\omega_{0}=\right.$ $\left.\sqrt{ } G_{m 1} \cdot G_{m 2} / \sqrt{ } C_{m} \cdot C_{o}\right)$ and quality factor $\left(\mathrm{Q}=\sqrt{ } G_{m 1} / G_{m 2}\right.$ $\left.\sqrt{ } C_{o} / C_{m}\right)$. With this modification, the positive and negative inputs of each transconductor would receive signals that have similar amplitude and phase. This means that the total voltage swing at the input of each transconductor will be reduced considerably compared to the conventional topology. Thus, the proposed topology relieves the need for a high-swing and linear transconductor since the total differential voltage swing at the input of each transconductor is much smaller than in the conventional configuration. Fig. $2 a$ shows the simulated transient response of each node in a biquadratic filter indicating the relative voltage swing and phase at different nodes. Note that the total voltage swing at the input of each transconductor remains always less that the corresponding voltage swing in the conventional topology.
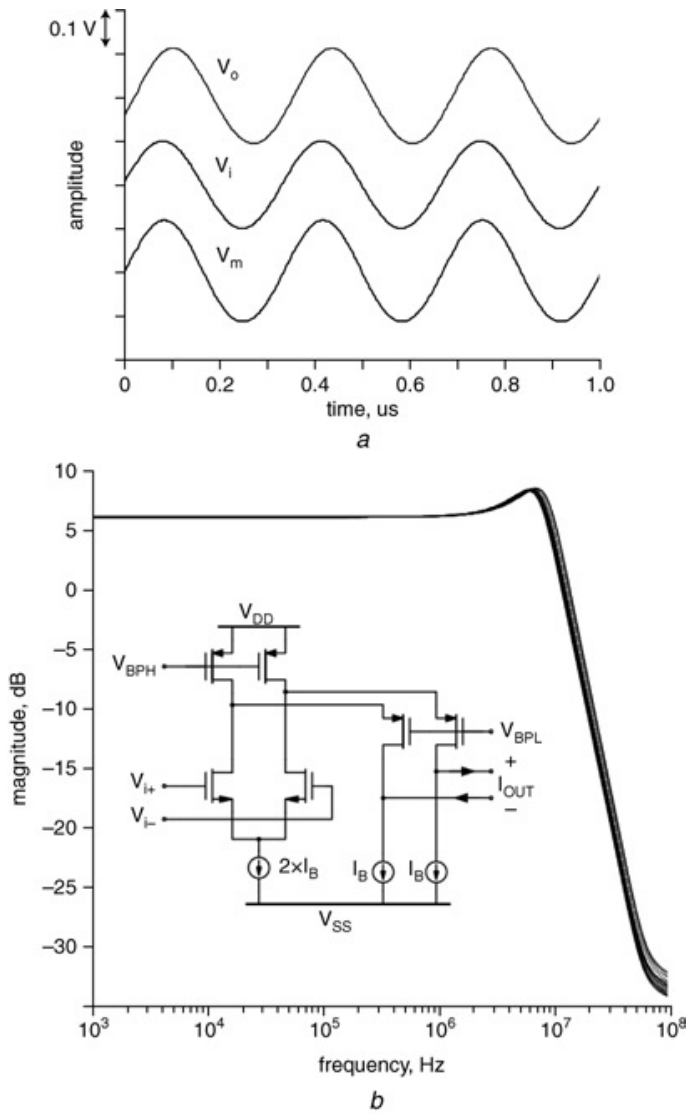

Fig. 2 Simulated transient waveforms at each node of biquadratic filter, and schematic diagram of differential folded cascode operational transconductor amplifier (OTA) and frequency response of proposed secondorder filter based on Monte Carlo simulations

a Simulated transient waveforms

$b$ Schematic diagram of differential folded cascode OTA and frequency response of proposed second-order filter based on Monte Carlo simulations

Simulation results: A second-order biquadratic filter has been designed with a conventional $0.18 \mu \mathrm{m}$ CMOS technology. A simple differential pair circuit with folded cascode output stage is used as the transconductor cell (Fig. 2b, inset) [2]. The entire biquadratic filter draws only $200 \mu \mathrm{A}$ from $1.8 \mathrm{~V}$ supply to implement a $10.7 \mathrm{MHz}$ 
cutoff frequency lowpass filter. Fig. $2 b$ shows the frequency response of the proposed second-order filter. Monte Carlo simulations show that the cutoff frequency variation of this filter exhibits $\sigma_{\mathrm{BW}}=5.9 \%$ in the presence of process variations and device mismatches.

Fig. 3 compares the total harmonic distortion (THD) of these two topologies. As shown in this plot, the THD of the proposed filter topology is about $30 \mathrm{~dB}$ better than the conventional topology, for input signals as high as $0 \mathrm{~dB}\left(2_{\mathrm{Vpp}, \mathrm{diff}}\right)$. Monte Carlo simulations show that the total output referred noise for both circuits is about $115 \mu \mathrm{V}_{\mathrm{rms}}$ and the DC common-mode gain remains less than $-41 \mathrm{~dB}$, proving the low sensitivity of the proposed filter topology to the common-mode signals.

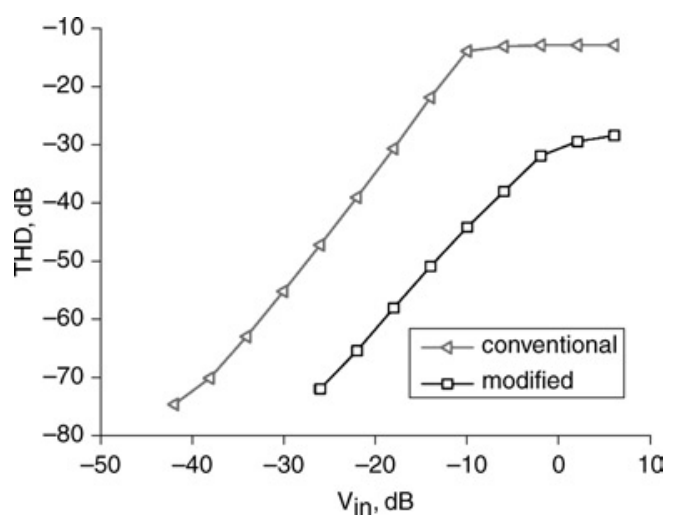

Fig. 3 Simulated total harmonic distortion of proposed filter topology compared to conventional topology $\left(f_{-3 d B}=10.7 \mathrm{MHz}, f_{\text {in }}=3 \mathrm{MHz}\right)$

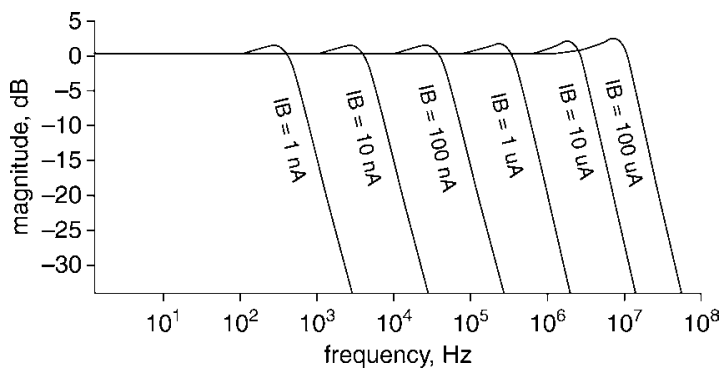

Fig. 4 Frequency response of biquadratic filter at different bias current values
Tuning range: Both of the topologies shown in Fig. 1 have a very wide frequency tuning range. Fig. 4 shows the frequency response for the proposed filter for different bias current values $\left(\mathrm{I}_{\mathrm{B}}\right)$. Because of the superior linearity performance of the proposed filter, this topology can be also considered a suitable candidate for implementing linear and wide tuning range continuous-time filters. Simulations show that the THD degrades by only $5 \mathrm{~dB}$ when the cutoff frequency of the filter is set to $400 \mathrm{~Hz}$ by reducing the bias current to $1 \mathrm{nA}$.

Conclusion: A modified topology for improving the linearity performance of biquadratic transconductor-C filters is proposed. This improvement is entirely based on a topology level modification, without any special requirement on transconductor specifications. Using a simple folded cascode transconductance amplifier as the basic transconductor circuit, simulations show that the linearity can be improved by at least $30 \mathrm{~dB}$ compared to the conventional topology. Relieving the need for linear transconductors, this approach represents a very effective solution for implementing high performance and wide tuning range filters with very low power consumption.

(C) The Institution of Engineering and Technology 2007 19 September 2007

Electronics Letters online no: 20072537

doi: 10.1049/el:20072537

A. Tajalli and Y. Leblebici (Microelectronic Systems Laboratory (LSM), Swiss Federal Institute of Technology (EPFL), 1015, Lausanne, Switzerland)

E-mail: armin.tajalli@epfl.ch

\section{References}

1 Franca, J.E., and Tsividis, Y.: 'Design of analog-digital VLSI circuits for telecommunications and signal processing' (Prentice Hall, 1993, 2nd edn.)

2 Johns, D.A., and Martin, K.: 'Analog integrated circuit design' (John Wiley \& Sons Inc., 1997)

3 Schaumann, R., and Van Valkenburg, V.: 'Design of analog filters' (Oxford University Press, 2001)

4 Calvo, B., et al.: 'Low-voltage pseudo-differential transconductor with improved tunability-linearity trade-off', Electron. Lett., 2006, 42, (15), pp. $862-863$ 